\title{
On the Properties of Materials for Designing Filters at Optical Frequencies
}

\author{
E. Topsakal and J.L. Volakis
}

This report was prepared as an account of work sponsored by the United States Government. Neither the United States, nor the United States Department of Energy, nor any of their employees, nor any of their contractors, subcontractors, or their employees, makes any warranty, express or implied, or assumes any legal liability or responsibility for the accuracy, completeness or usefulness of any information, apparatus, product or process disclosed, or represents that its use would not infringe privately owned rights. 


\title{
On the Properties of Materials for Designing Filters at Optical Frequencies
}

\author{
'E. Topsakal and ${ }^{1,2}$ J.L. Volakis \\ 'The University of Michigan, Ann Arbor, Michigan 48109-2122 \\ ${ }^{2}$ The Ohio State University, Columbus, OH 43210 \\ \{lopsakal,volakis?@umich.edu
}

\section{Introduction}

Frequency Selective Surfaces/Volumes (FSS/Vs), periodic structures with frequency selective properties, have widely been used for millimeter and microwave applicarions [1]. Some applications include filters (band pass,-band stop), reflectors, radoms etc. FSS/Ns typically consist of a single or multiple material layers. Multiple layers (with each layer having a different frequency selectivity) are used for broadband applications. In recent years there has been an interest in using these structures at optical wavelengths. One of the applications is in thermophotovoltaic filters used to convert thermal energy into electricity. The filter is designed to transmit those wavelengths that can be efficiently converted into electricity, and 10 reflect other spectra, which leads to energy conservation and an increase in overall system efficiency. These filters can be used in space missions to help decrease energy consumption and reduce spacecrafi mass, cost, and fuel loading.

Numerical simulations of such filters are very limited in the literature [2,3]. Existing modeling approaches are based on the assumption of purely metallic (perfectly conducting) structures on substrates. However, in practice, metals have finite conductivity that can lead to power absorption in the metal. At optical frequencies the usual material properties and perfect electric conductor (PEC) assumption is not applicable. Moreover, the conventional methods, such as using resistive sheets or lossy dielectrics to simulate metallic losses, are not accurate. Our goal is to provide a new approach for modeling metallic losses more accurately at the optical frequencies.

\section{Modcls for simulating metallic structures}

Several models exist and have been discussed in the literature to model metallic structures in numerical simulations. These models are PEC, the R-Card (Resistive Sheet) and Lossy Dielectric models. For non-PEC surfaces the resistive sheet model is the most popular. In the limiting case resistive sheet reduces to $P E C$ via

$$
R=\frac{1}{\sigma \tau} \Rightarrow \sigma=\infty(P E C) \Rightarrow R=0
$$

In the case of Aluminum,

$$
\left.R=\frac{1}{\sigma} \Rightarrow \sigma=38000000 \text { (Alu } \min u m\right), t=0.09 \mu \Rightarrow R=0.292 \Omega \text {. }
$$

Resistive model is efficient for material thicknesses that are small compared to the wavelcngth of operation $(\angle \lambda / 20)$. When the thickness is increased this model fails (especially for the oblique incidence). This is the case at the optical frequencies. When the lossy dielectric model is used, the metallic regions are modeled using high loss dielectrics and a relative dielectric permittivity calculated via the formula,

$$
\varepsilon=\varepsilon_{0} \varepsilon_{\mathrm{r}}=\varepsilon_{0}\left(1-j \sigma / \omega \varepsilon_{0}\right) \Rightarrow \varepsilon_{\mathrm{r}}=1-\mathrm{j} \sigma / \omega \varepsilon_{0}
$$


Here $\varepsilon_{0}$ is the dielectric constant of free space, $\sigma$ is the conductivity and $\omega$ is the operating frequency. The lossy dielectric model is better than the resistive model since it simulates the finite conductivity in a volumetric manner. Nevertheless, the lossy dielectric model is not valid above 15THz. Thus at optical frequencies it is necessary to consider alternative models as discussed next.

\section{Matcrial Modeling at the optical frequencies}

Consider a homogenous isotropic medium of relative dielectric constant $\varepsilon$, permeability $\mu$ and conductivity $\sigma$. From Maxwell's equation in a source free region,

$$
\Delta E+k^{2} E=0 ; \quad \Delta H+k^{2} H=0
$$

where

$$
k^{2}=\omega^{2} \mu\left(\varepsilon-j \frac{\sigma}{\omega}\right) / c^{2}, \quad\left(c=1 / \sqrt{\varepsilon_{0} \mu_{0}}\right) .
$$

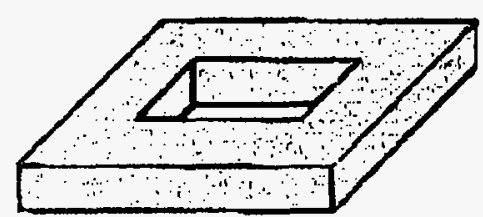

a) Unit Cell

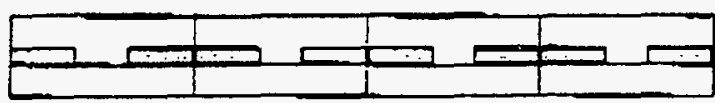

b) Commensurate Array

Figure.1 Band Pass Filter Geometry

Note that $k^{2}$ is identical with the corresponding equations for non-conducting media when $\varepsilon$ is replaced by

$$
\hat{\varepsilon}=\varepsilon-j \frac{\sigma}{\omega}
$$

Correspondingly, we can define complex velocity and the refractive index of the nedium viz.

$$
\hat{v}=\frac{c}{\sqrt{\mu \hat{\varepsilon}}} ; \quad \hat{n}=\frac{c}{\hat{v}}=n-j \psi
$$

Here $n$ and $\psi$ are real and are the standard refraction index and attenuation constant, respectively. They can be expressed in terms of the material constants $\varepsilon, \mu$ and $\sigma$ as

$$
\begin{aligned}
& \hat{n}^{2}=n^{2}-j 2 n \psi-\psi^{2} \\
& \hat{n}^{2}=\mu \hat{\varepsilon}=\mu\left(\varepsilon-j \frac{\sigma}{\omega}\right) .
\end{aligned}
$$

For sufficiently low frequencies $\sigma$ is real. However there is a general consensus [4] that this is true only for wavelengths greater than $\lambda>2 \times 10^{-3} \mathrm{~cm}(f<15 \mathrm{THz}$ ). Classical expressions for the value of $\sigma$ will not work at frequencies greater than $15 \mathrm{THz}$. To generate mathematical models of a thick conductor it is necessary to provide proper expressions of values of $\sigma$. Thus to find $\sigma$ we begin with the equation

$$
m \frac{d^{2} \mathbf{r}}{d t^{2}}+m \beta \frac{d \mathbf{r}}{d t}=e \mathbf{E}
$$




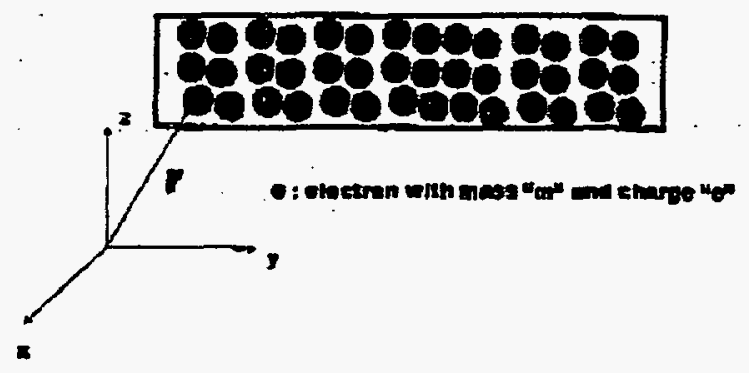

Figure 2. Metallic structure at the optical frequencies

$m$ is the mass, $e$ is the electron charge and $\beta$ is the dumping constant $\left(-10^{14}\right.$ for metals). The solution of (9) can be given as

$$
\mathbf{r}=-\frac{e}{m\left(\omega^{2}+i \beta \omega\right)} \mathbf{E} .
$$

This refers to a periodic motion and gives rise to a current in the medium. If there are " $N$ " free electrons per unit volume, the current density $J$ is given by

$$
\mathbf{J}=N e \frac{d \mathbf{r}}{d t}=\frac{N e^{2}}{m(\beta-i \omega)} \mathbf{E} .
$$

Comparing ( 11 ) with $J=\sigma E$, we have

$$
\sigma=\frac{N e^{2}}{m(\beta-i \omega)} .
$$

It is clear from (12) that when $\omega<<\beta, \sigma$ can be approximated by $\sigma_{0}=\mathrm{Ne} / \mathrm{m} \beta$ which is real. However for $\omega>>\beta$ (which is the case at optical frequencies) the imaginary part of $\sigma$ becomes large compared to its real part. If we use this expression for $\sigma$, then $\hat{\varepsilon}$ in (8b) takes the form

$$
\begin{aligned}
& \operatorname{Re}\{\hat{\varepsilon}\}=\varepsilon^{\prime}=n^{2}-\psi^{2}=1-\frac{4 \pi N e^{2}}{m\left(\omega^{2}+\beta^{2}\right)} \\
& \operatorname{Im}\{\hat{\varepsilon}\}=\varepsilon^{\prime \prime}=2 n \psi=\frac{2 \pi N e^{2} / \beta}{m \omega\left(\omega^{2}+\beta^{2}\right)} .
\end{aligned}
$$

The dielectric constant at the desired frequencies can then be found using (13a) and (13b) or by using available measured data for $\mathfrak{n}$ and $\psi[5]$.

\section{Results/ Conclusions}

As an example, we consider the commensurate array of the slot FSVs shown in Fig. 4 and employ various models for its analysis. The geometry refers to a unit cell size $700 \times 700 \mathrm{~nm}^{2}$, consisting of two metallic regions; the outer ring is $50 \mathrm{~nm}$ wide, and the inner block is $400 \times 400 \mathrm{~nm}^{2}$. The thickness of the unit cell is $90 \mathrm{~nm}$ and the analysis done using a finite element boundary integral code [3]. Clearly the models mentioned above give different answers and vary in the amount of absorption (see fig. S). Specifically the resistive model does not provide much absorption and is also showing a shifi due to modeling the actual thick structure with a sheet. The LD model is better but it over predicts the losses. The new model predicts a $\sim 10 \%$ loss and measurements are needed to verify it. In figure 6 the transmitted, reflected and absorbed power results are presented for the proposed model. Note that the power loss observed in transmission is due to the absorption in the filter. 


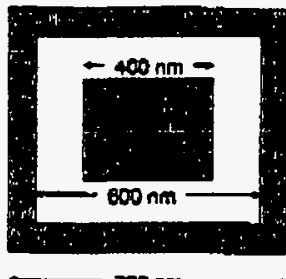

Top Vew
$90 \mathrm{~nm}$ !

Slde Viow

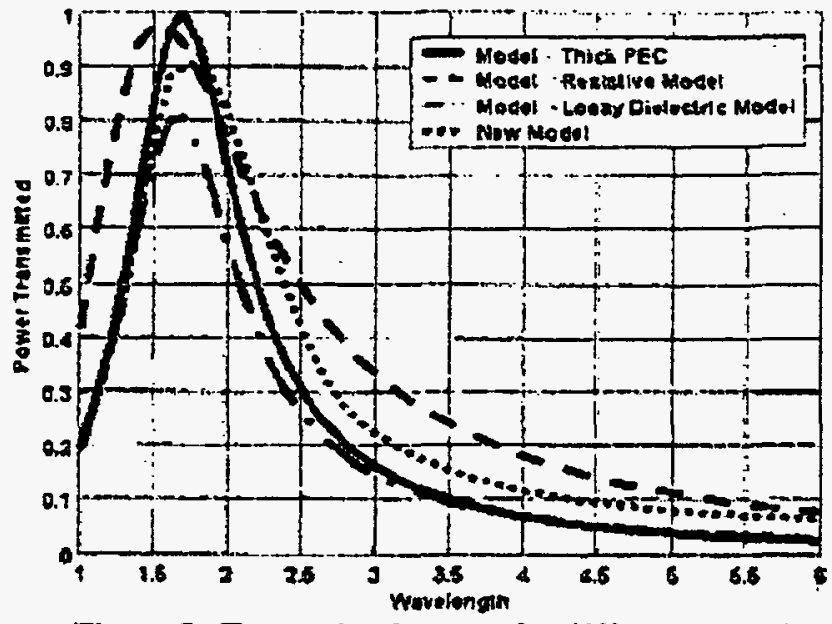

Figure 5. Transmitted power for different models

Figure 4. FSV Gcometry

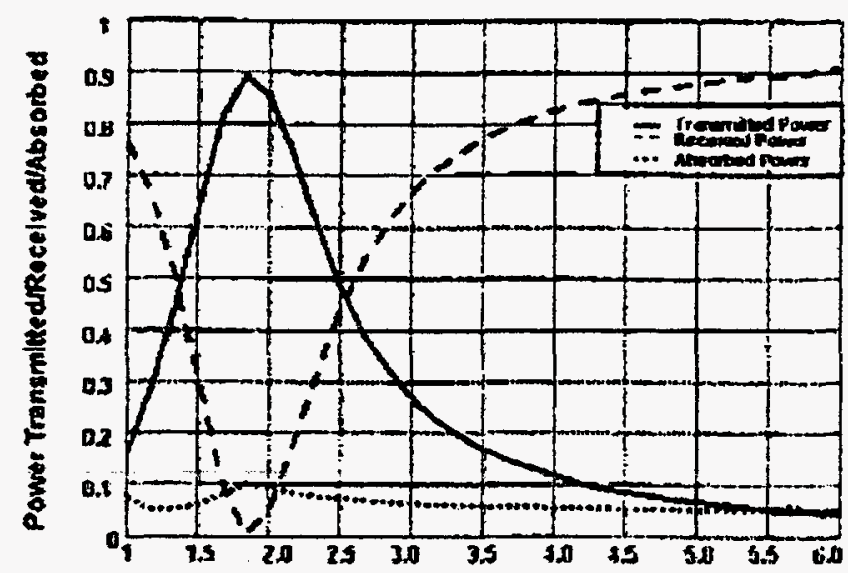

Figure 6. Transmitted/Received and Absorbed power for proposed model

\section{References}

[1] B. Munk, "Frequency Selective Surfaces: Theory and Design", John Wiley Pub, 2000.

[2] T.K. Wu, "Infrared Filters For High Frequency Thermovoltaic Devices", Microwave and Optical Technology letters, vol.15, no.1, May 1997.

[3] T.F. Eibert, Erdemli Y., J.L. Volakis, "Upgrading the Hybrid Finite Element - Boundary Integral Method Using Multilayer Periodic Green's Functions", IEEE Antennas and Propagation Society Intermational Symposium, 2002, pp:282-284.

[4] M. Born and Wolf E., "Principle of Optics", Cambridge University Press, 7" edition 2002.

[5] E.D. Palik, "Handbook of Oprical Conslants of Solids", Academic Press, 1985. 